\title{
Long non-coding RNA SPRY4-IT1 promotes the proliferation and invasion of U251 cells through upregulation of SKA2
}

\author{
XIAO-JUN HE ${ }^{1,2^{*}}$, ER-BAO BIAN ${ }^{1,2^{*}}$, CHUN-CHUN MA ${ }^{1,2}$, CHAO WANG $^{1,2}$, \\ HONG-LIANG WANG ${ }^{1,2}$ and BING ZHAO ${ }^{1,2}$ \\ ${ }^{1}$ Department of Neurosurgery, The Second Affiliated Hospital of Anhui Medical University; \\ ${ }^{2}$ Cerebral Vascular Disease Research Center, Anhui Medical University, Hefei, Anhui 230601, P.R. China
}

Received October 20, 2015; Accepted November 8, 2017

DOI: $10.3892 / \mathrm{ol} .2018 .7776$

\begin{abstract}
The long non-coding RNA SPRY4-intronic transcript 1 (SPRY4-IT1) has been shown to promote the progression of cancer; however, the role of SPRY4-IT1 in glioma remains unclear. The present study demonstrated that SPRY4-IT1 expression was markedly increased in glioma tissues and cells compared with normal brain tissues, whereas knockdown of SPRY4-IT1 inhibited cell proliferation, migration, and invasion in U251 cells. Spindle and kinetochore associated complex subunit 2 (SKA2) was found to be a target of SPRY4-IT1 and was downregulated by SPRY4-IT1-knockdown. Additionally, SPRY4-IT1 expression was positively correlated with SKA2 in glioma tissues. To the best of our knowledge, the present study provides the first demonstration that SKA2 may have an oncogenic role in U251 cells. These results indicate that SPRY4-IT1 may serve a notable role in the molecular etiology of glioma and represents a potential target in glioma therapy.
\end{abstract}

\section{Introduction}

Gliomas are the most common form of central nervous system (CNS) primary tumors, accounting for $>50 \%$ of all primary CNS tumors (1). Gliomas are characterized by increased proliferation, invasion and malignancy. Despite the use of

Correspondence to: Professor Bing Zhao, Department of Neurosurgery, The Second Affiliated Hospital of Anhui Medical University, 678 Fu Rong Road, Hefei, Anhui 230601, P.R. China

E-mail: aydzhb@126.com

*Contributed equally

Abbreviations: CNS, central nervous system; lncRNA, long non-coding RNAs; SKA2, spindle and kinetochore associated complex subunit 2; DMEM, Dulbecco's modified Eagle's medium; FBS, fetal bovine serum; SPRY4-IT1, SPRY4-intronic transcript 1; $\mathrm{NC}$, negative control

Key words: astrocytoma, long non-coding RNAs, SPRY4-intronic transcript 1 , spindle and kinetochore associated complex subunit 2 aggressive therapeutic modalities, including surgical resection, radiation and chemotherapy, the prognosis for patients with malignant gliomas remains poor (2-4). Advances in increasing the survival rate of glioma patients have been limited, as the pathophysiological mechanisms of glioma remains unclear. Therefore, a deeper understanding of the pathways involved in the development of glioma is required to develop a novel therapeutic target for glioma.

Recent improvements in high-resolution microarray and genome-wide sequencing analysis have revealed that human genome includes $\sim 20,000$ protein-coding genes, however, most of the human genome is pervasively transcribed into ncRNA $(5,6)$. ncRNAs are divided into two groups, according to their size, long ncRNAs (lncRNA) and small ncRNAs. Of the ncRNAs, microRNAs have attracted considerable attention as they serve a number of pivotal roles in cancer via the silencing of target genes $(7,8)$. However, the field of lncRNAs is an emerging area in ncRNAs study. IncRNAs have been reported to regulate a wide of biological processes as they are involved in every step of mRNA biology, including transcription, mRNA splicing, RNA decay and translation (9-11).

lncRNAs are a class of non-coding RNA transcript $>200$ nucleotides in length that have no protein-coding potential. Recent studies have demonstrated that lncRNAs serve a vital role in epigenetic modification. IncRNAs participate in various biological and pathological processes by controlling gene expression via diverse mechanisms including transcription, post-transcriptional processing, genomic imprinting, chromatin modification and the regulation of protein function (12-14). For example, Wang et al (15) demonstrated that the ectopic expression of maternally expressed 3 (MEG3), a lncRNA that is markedly downregulated in astrocytoma, could inhibit cell proliferation and promote cell apoptosis in astrocytoma cells, indicating the potential tumor-suppressive function of MEG3. In addition, the expression of HOX transcript antisense RNA (HOTAIR) was closely associated with glioma grade and poor prognosis, and an independent prognostic factor in GBM patients (16). Knockdown of HOTAIR inhibited colony formation and cell cycle $\mathrm{G}_{0} / \mathrm{G}_{1}$ arrest (16). These findings indicate that lncRNAs are involved in the development of gliomas. IncRNA SPRY4-intronic transcript 1 (SPRY4-IT1) is a 687-nucleotide unspliced, polyadenylated transcript that is transcribed from the second intron of the SPRY4 gene. The 
upregulation of SPRY4-IT1 expression has been observed in melanoma cells, whereas the knockdown of SPRY4-IT1 inhibited invasion, inducing cell growth arrest and apoptosis (17). However, the role of lncRNA SPRY4-IT1 in glioma is unclear.

Spindle and kinetochore associated complex subunit 2 (SKA2) is encoded by an 831-nucleotide cDNA sequence and is located on human chromosome 17q 23.2. SKA2 is required for the assembly of condensed chromosomes on the metaphase plate and is involved in the maintenance of the metaphase plate and/or spindle checkpoint silencing (18). Rice et al (19) demonstrated that the enforced expression of SKA2 induced glucocorticoid transactivation in HepG2 cells, whereas knockdown of SKA2 in A549 human lung epithelial cells reduced transactivation and suppressed dexamethasone inhibition of proliferation.

The objective of the present study was to explore the role of SPRY4-IT1 in glioma. SPRY4-IT1 expression was examined in glioma tissues and U251 cell lines using reverse transcription-quantitative polymerase chain reaction (RT-qPCR). and the biological functions of SPRY4-IT1 in U251 cells. Additionally, it was observed that SKA2 was a downstream target gene of SPRY4-IT1 and that SKA2 promoted U251 cells proliferation and invasion. The present study promotes the understanding of the role of SPRY4-IT1 as regulators of glioma pathogenesis, and contributes to the development of lncRNA-directed diagnostics and therapeutics.

\section{Materials and methods}

Materials. Fetal bovine serum (FBS) and Dulbecco's modified Eagle's medium (DMEM) were purchased from Hyclone; GE Healthcare Life Sciences (Logan, UT, USA). Lipofectamine 2000 and TRIzol reagent was purchased from Invitrogen; Thermo Fisher Scientific, Inc. (Waltham, MA, USA). M-MLV Reverse Transcriptase was obtained from Promega Corporation (Madison, WI, USA). All other chemicals were purchased from Sigma-Aldrich; Merck KGaA (Darmstadt, Germany). The antibodies used were as follows: Anti-proliferating cell nuclear antigen (PCNA; cat. no. bs-0754R), anti-cyclin D1 (cat. no. bs-0623R); anti-matrix metalloproteinase-2 (MMP2; cat. no. bs-4605R); and anti-MMP9 (1:400 dilution, cat. no. bs-4593R; BIOSS, Beijing, China), anti- $\beta$-actin (cat. no. ab8226), anti-SKA2 (cat. no. ab91551) (1:1,000 dilution; Abcam, Cambridge, UK).

Patients and tissue samples. Tissue samples from glioma tumors and normal brain tissues were collected from the neurosurgery department of the Second Affiliated Hospital of Anhui Medical University (Hefei, China) between May 2014 and March 2015. Samples were collected and preserved at $-80^{\circ} \mathrm{C}$ and their histological type was further confirmed according to the World Health Organization (WHO) criteria. A total of 64 glioma samples (WHO I/II, n=27 WHO III/IV, $\mathrm{n}=37)$ and normal brain tissues $(\mathrm{n}=9)$ were used in the present study (20). Patients selected included 50 males and 23 females, with an age range between 16 and 64 years (median age, 46 years). The present study was approved by the Biomedical Ethics Committee of Anhui Medical University and patients provided written informed consent.
Cell culture procedures. Human astrocytoma U251 cells were purchased from the American Type Culture Collection (ATCC; Manassas, VA, USA). Cells were cultured in DMEM, supplemented with $10 \%$ heat-inactivated FBS and $100 \mathrm{U} / \mathrm{ml}$ penicillin/streptomycin (Thermo Fisher Scientific, Inc.). Cells cultures were maintained at $37^{\circ} \mathrm{C}$ in humidified atmosphere of $5 \% \mathrm{CO}_{2}$. Small interfering RNAs (siRNAs) were chemically synthesized by GenePharma (Shanghai, China). The sequences are: SPRY4-IT1 siRNA, GCTTTCTGATTC CAAGGCCTATTAA; SKA2 siRNA, AAGAAATCAAGA CTAATCATCTT; Si-NC, UUCUCCGAACGUGUCACG UTT. U251 cells $\left(2 \times 10^{5}\right)$ were transfected with siRNA using Lipofectamine 2000 transfection reagent (Life Technologies; Thermo Fisher Scientific, Inc.) according to the manufacturer's instructions. After $48 \mathrm{~h}$, cells transfected with siRNA were harvested for RT-qPCR to determine the transfection efficiency.

RT-qPCR analysis. Total RNA was extracted from U251 cells and patient samples using TRIzol reagent. The first-strand cDNA was synthesized from total RNA using the Thermoscript RT-PCR system (Thermo Fisher Scientific, Inc.) at $65^{\circ} \mathrm{C}$ for 5 min. RT-qPCR analysis was performed using SYBR Green Master Mix kit on Thermo Fisher connect Real-Time PCR platform (Thermo Fisher Scientific, Inc.). In brief, each PCR reaction mixture containing $10 \mu \mathrm{l}$ of $2 \mathrm{X}$ SYBR GreenMaster Mix, $1 \mu \mathrm{l}$ of sense and antisense primers $(5 \mu \mathrm{mol} / \mu \mathrm{l})$ and $1 \mu \mathrm{l}$ of cDNA (10 ng), was run for 45 cycles with denaturation at $95^{\circ} \mathrm{C}$ for $15 \mathrm{sec}$, annealing at $60^{\circ} \mathrm{C}$ for $30 \mathrm{sec}$ and extension at $72^{\circ} \mathrm{C}$ for $30 \mathrm{sec}$ in a total volume of $20 \mu \mathrm{l}$. For relative quantification, $2^{-\Delta \Delta \mathrm{Cq}}$ was calculated and used as an indication of the relative expression levels (21), which was calculated by subtracting quantification cycle $(\mathrm{Cq})$ values of the control gene from the Cq values of SPRY4-IT1. The primer sequences were as follows: SPRY4-IT1 forward, 5'-AGCCACATAAATTCA GCAGA-3' and reverse, 5'-CGATGTAGTAGGATTCCTTTC A-3'; SKA2 forward, 5'-CCGCTTTAAACCAGTTGCTG-3' and reverse, 5'-CTCTGCCGCAGTTTTCTCTT-3'. GAPDH was applied as an internal control. The primer sequences of GAPDH were: Forward, 5'-AGCAAGAGCACAAGAGGA AG-3' and reverse, 5'-GGTTGAGCACAGGGTACTTT-3'.

MTT assay. U251 cells were trypsinized, resuspended and seeded into 96-well plates at a concentration of 2,000 cells/ well, and incubated at $37^{\circ} \mathrm{C} 48 \mathrm{~h}$ after siRNA treatment. The number of viable cells was measured at daily intervals $(0,12$, 24, and $48 \mathrm{~h}$ ). At each time-point, $10 \mu \mathrm{l}$ of $5 \mathrm{mg} / \mathrm{ml} \mathrm{MTT}$ (Beijing Dingguo Biotechnology Co., Ltd., Beijing, China) was added and incubated for $4 \mathrm{~h}$. Then the medium was removed carefully and $100 \mu \mathrm{l}$ dimethyl sulfoxide was added at the end of incubation. The absorbance was measured at $592 \mathrm{~nm}$ on the spectrophotometer.

Colony formation assay. A total of 200 U251 cells were seeded in 6-well plates following a 48-h siRNA treatment. The medium was changed at regular time intervals. After 7 days of culture at $37^{\circ} \mathrm{C}$, the natural colonies were washed with PBS and fixed with $4 \%$ paraformaldehyde for $30 \mathrm{~min}$ at room temperature. The colonies were then stained with methylene blue $(1 \%)$ for $10 \mathrm{~min}$ at room temperature, washed 
with water and air-dried. The total number of colonies with more than 50 cells was counted using a fluorescent microscope (x100 magnification).

Scratch wound assay. U251 astrocytoma cells were transfected with siRNA targeted at SPRY4-IT1 (si-SPRY4-IT1) or si-negative control (si-NC). Cells $\left(5 \times 10^{5}\right)$ were cultured in 6 -well plates. Wounds were created in adherent cells using a $20 \mu \mathrm{l}$ pipette tip at $48 \mathrm{~h}$ after transfection. The cells were then washed three times with PBS to remove any free-floating cells and debris. Serum-free DMEM was added, and the cells were incubated under normal conditions. Wound healing was observed after $24 \mathrm{~h}$ using a light microscope. Images of representative scratch lines were captured using digital microscopy once culture inserts were removed. Each experiment was repeated in triplicate.

In vitro migration and invasion assays. U251 cells $\left(5 \times 10^{5}\right)$ were transfected with si-SPRY4-IT1 after $48 \mathrm{~h}$, and then plated into the upper chamber of polycarbonate transwell filters (without Matrigel for the transwell assay) or plated on the top side of polycarbonate transwell filter coated with Matrigel (for the invasion assay) in the upper chamber of the QCM $^{\text {TM }}$ 24-Well Cell Invasion Assay (Cell Biolabs, Inc., Sand Diego, CA, USA). For transwell migration assays, cells were suspended in $150 \mu \mathrm{l}$ DMEM without serum, and DMEM supplemented with $10 \%$ FBS was used in the lower chamber. For the invasion assay, cells were suspended in medium without serum, and DMEM supplemented with $10 \%$ FBS was used as a chemoattractant in the lower chamber. The cells were incubated at $37^{\circ} \mathrm{C}$ for $24 \mathrm{~h}$ for each assay. The non-migratory or non-invasive cells in the top chambers were removed with cotton swabs. The migrated and invaded cells on the lower membrane surface were fixed with methanol and stained with crystal violet. Cells were counted visually in 5 random fields using a light microscope (x100). In addition, migrated and invaded cells were dissociated, lysed and quantified at $570 \mathrm{~nm}$ using spectrophotometer.

Western blotting. U251 astrocytoma cells transfected with si-SPRY4-IT1 were lysed with RIPA lysis buffer (Beyotime Institute of Biotechnology, Haimen, China). Whole extracts were prepared, and protein concentrations were determined using the BCA protein assay kit (Boster Biological Technology, Pleasanton, CA, USA). Whole-cell extracts (20 or $40 \mu \mathrm{g}$ ) were then fractionated using 8 or $12 \%$ SDS-PAGE. Gels were run at a $120 \mathrm{~V}$ for $2 \mathrm{~h}$ then transfer onto a polyvinylidene fluoride membrane (EMD Millipore, Billerica, MA, USA). Nitrocellulose blots were incubated for $1 \mathrm{~h}$ with primary antibodies diluted in TBS/Tween-20 (0.075\% Tween-20) containing 3\% skimmed milk powder. Antibody was diluted. Following incubation with the afore mentioned primary antibodies at $4^{\circ} \mathrm{C}$ overnight, blots were washed three times in TBS/Tween-20 prior to incubation at room temperature for $1 \mathrm{~h}$ with goat anti-mouse (cat. no. ZB-2305) or anti-rabbit (cat. no. ZB-2301) (both from ZSGB-BIO; Origene, Beijing, China) horseradish peroxidase conjugated antibody at a 1:10,000 dilution in TBS/Tween-20 containing 5\% milk. Following extensive washing in TBS/Tween-20, the blots were rinsed with distilled water and proteins were detected using the enhanced chemiluminescence system. Proteins were visualized with an enhanced chemiluminescence kit (ECL-plus; Thermo Fisher Scientific, Inc.). The quantification of the bands was performed using ImageJ software (version 1.48; National Institutes of Health, Bethesda, MD, USA).

Statistical analysis. All data were expressed as mean \pm standard deviation of three independent experiments, in which each assay was performed in triplicate. Data were analyzed with SPSS 16.0 software (SPSS, Inc., Chicago, IL, USA). Evaluation of the data was performed by t-test (two-sided) or one-way analysis of variance, followed by Tukey's post hoc test. Pearson's test was performed to calculate the association between SPRY-IT1 and SKA2 expression. $\mathrm{P}<0.05$ was considered to indicate a statistically significant difference.

\section{Results}

Expression of SPRY4-IT1 was upregulated in glioma. To determine the association between SPRY4-IT1 expression and glioma with different degrees, RT-qPCR was performed to evaluate the expression levels of SPRY4-IT1 in 9 normal brain tissue samples and 64 freshly dissected glioma samples. As shown in Fig. 1A, levels of SPRY4-IT1 were upregulated in gliomas tissues compared with normal brain tissues. Additionally, expression of SPRY4-IT1 was markedly upregulated in high-grade glioma samples (WHO tumor grades III and IV) and, to a lesser degree, increased in WHO tumor grades I and II glioma samples, compared with normal brain tissues (Fig. 1B). SPRY4-IT1 expression was also examined in U251 cell lines and normal brain tissues. As shown in Fig. 1C, SPRY4-IT1 expression was higher in U251 cell lines than in normal brain tissues. Therefore, these data clearly indicate that high levels of SPRY4-IT1 expression are present in glioma tissues and indicate that this high expression may contribute to glioma pathogenesis.

Knockdown of SPRY4-IT1 inhibited U251 cells proliferation. Astrocytoma is a glioma that originates from astrocytes (20). U251 astrocytoma cells are frequently used to study glioma malignant biological characteristics, so U251 cells were selected for use in the present study. To determine whether siRNAs were able to be transfected into U251 cells, cells transfected with si-SPRY4-IT1 and si-NC were assessed by RT-qPCR. Cells transfected with si-SPRY4-IT1 exhibited significantly lower expression of SPRY4-IT1 mRNA in astrocytoma cells compared with si-NC cells (Fig. 1D). To investigate the role of SPRY4-IT1 in U251 cells proliferation, an MTT assay was performed. Knockdown of SPRY4-IT1 in U251 cells markedly suppressed cellular proliferation (Fig. 2A). Similarly, the size of colonies formed by si-SPRY4-IT1 cells were markedly decreased in U251 cells compared with si-NC cells, indicating that the decrease of SPRY4-IT1 expression significantly inhibits colony formation by U251 cells (Fig. 2B).

SPRY4-IT1 knockdown suppressed U251 cells migration and invasion. To investigate whether SPRY4-IT1 serves a direct functional role in facilitating U251 cell migration, U251 cell migration was assessed using a scratch wound assay. Data from 

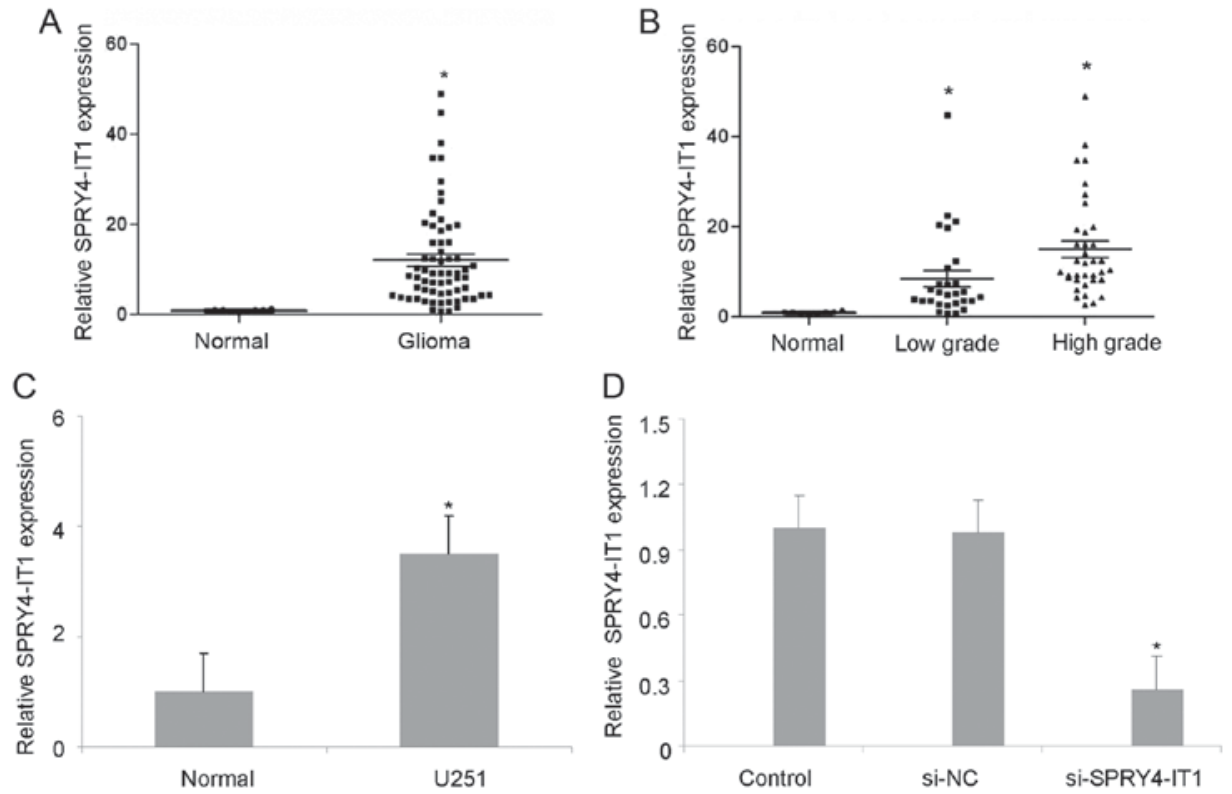

Figure 1. The expression of SPRY4-IT1 in human glioma tumor samples and cells lines. (A) RT-qPCR analysis of SPRY4-IT1 expression in glioma tumor samples and normal brain tissues. "P<0.01 vs. normal. (B) RT-qPCR analysis of SPRY4-IT1 expression in normal brain tissues, low-grade and high-grade glioma samples. "P<0.01 vs. normal. (C) RT-qPCR analysis of SPRY4-IT1 expression in normal brain tissues and U251 cells. "P<0.01 vs. normal. (D) RT-qPCR analysis of SPRY4-IT1 expression in si-SPRY4-IT1, si-NC and untransfected U251 control cells. "P<0.01 vs. si-NC. SPRY4-IT1, SPRY4-intronic transcript 1; si-NC, U251 cells transfected with negative control small interfering RNA.
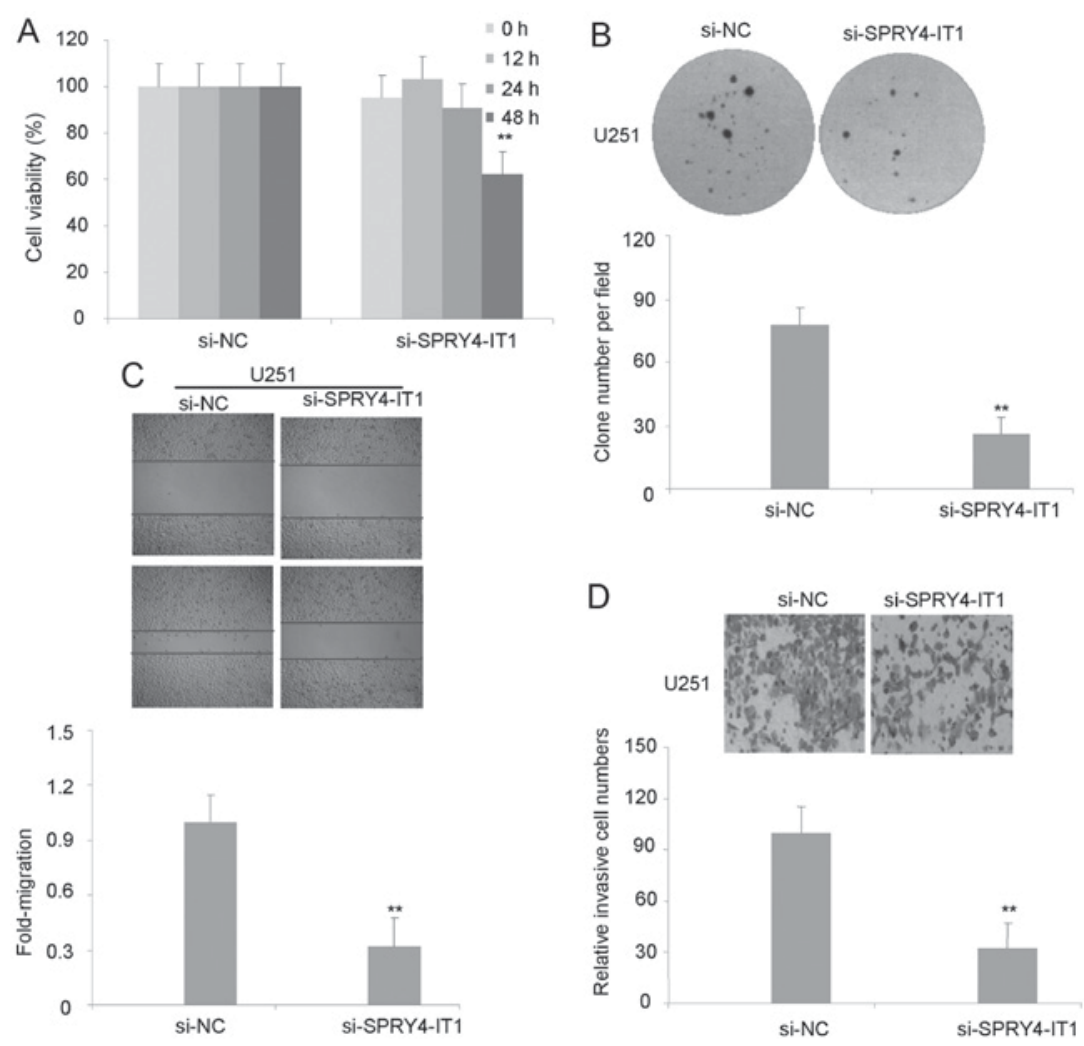

Figure 2. Knockdown of SPRY4-IT1 inhibits cellular proliferation, migration and invasion in U251 cells. (A) si-SPRY4-IT1 cell proliferation was significantly inhibited compared with si-NC cells, as detected by the MTT assay. (B) Effects of SPRY4-IT1 on clone formation in U251 cells. (C) Scratches were made on confluent monolayers si-NC or si-SPRY4-IT1 cells. (D) U251 cells infected with si-SPRY4-IT1 displayed significantly lower invasion capacity compared with those infected with si-NC. The widths of the gaps from 3 experiments were measured, the mean was calculated and the results presented in a bar graph. ${ }^{* *} \mathrm{P}<0.01$ vs. si-NC. SPRY4-IT1, SPRY4-intronic transcript 1; si-NC, U251 cells transfected with negative control small interfering RNA.

this assay revealed that transfection with si-SPRY4-IT1 but not si-NC reduced the cell migration of U251 cells (Fig. 2C).
As shown in Fig. 2D, invasion of U251 cells was reduced following inhibition of SPRY4-IT1. These results indicate that 

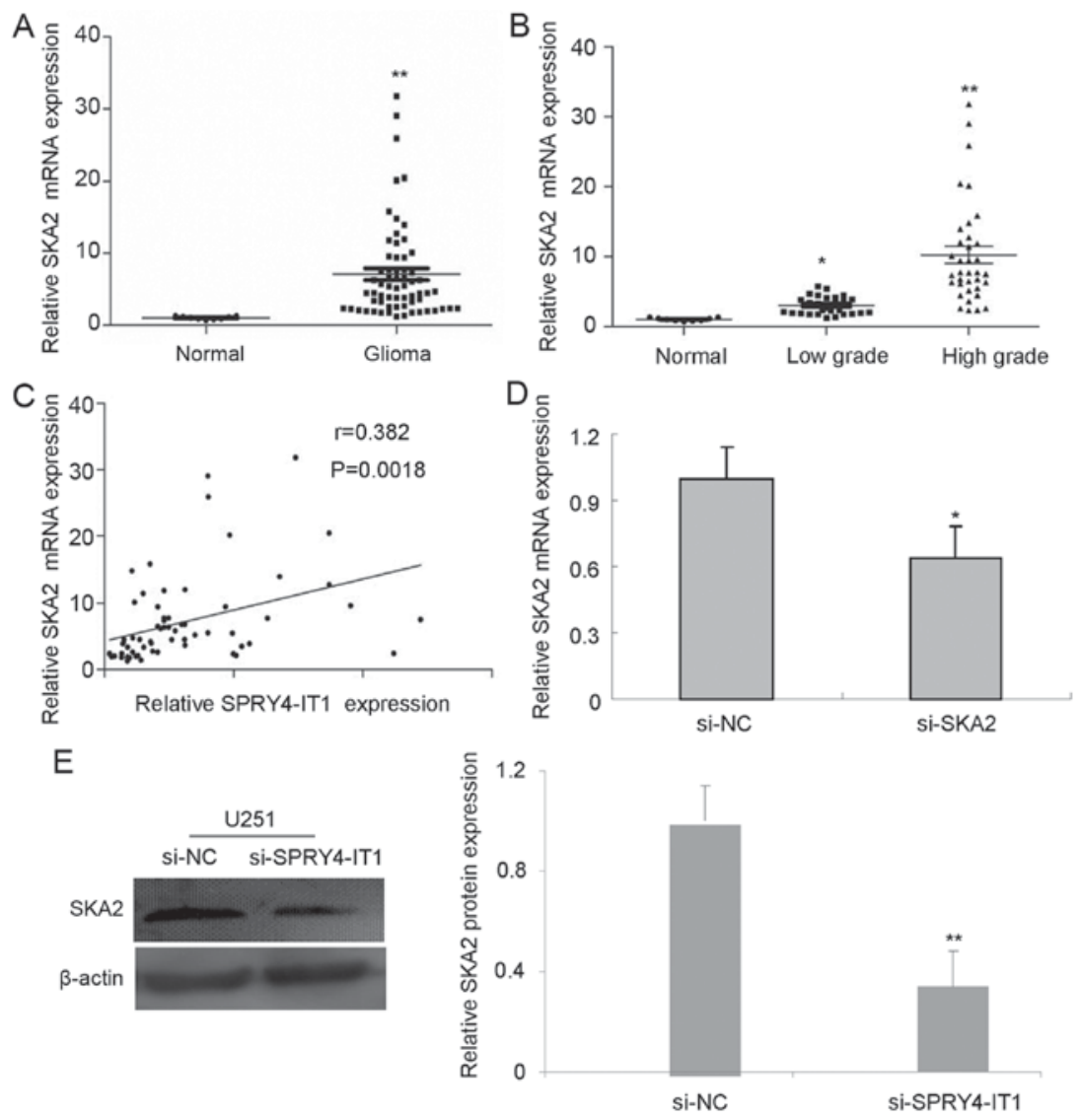

Figure 3. SPRY4-IT1 regulates the expression of SKA2. RT-qPCR analysis of SKA2 expression in (A) 64 glioma tissues and 9 normal brain tissues and (B) normal, low-grade and high-grade glioma samples. $* \mathrm{P}<0.05$, **P $<0.01$ vs. Normal. (C) SKA2 expression positively correlated with that of SPRY4-IT1 in 64 glioma samples. SKA2 and SPRY4-IT1 expression levels in these samples were measured by RT-qPCR, and data were subjected to Pearson's correlation analysis ( $r=0.382, \mathrm{P}=0.0018$; Pearson's correlation analysis). (D) Knockdown of SPRY4-IT1 decreases SKA2 mRNA expression compared with NC in U251 cells. (E) Knockdown of SPRY4-IT1 decreases SKA2 protein expression compared with NC in U251 cells. ${ }^{*} \mathrm{P}<0.05,{ }^{* *} \mathrm{P}<0.01$ vs. si-NC. RT-qPCR, reverse transcription-quantitative polymerase chain reaction; SKA2, spindle and kinetochore associated complex subunit 2; SPRY4-IT1, SPRY4-intronic transcript 1; si-NC, U251 cells transfected with negative control small interfering RNA.

SPRY4-IT1 can promote the U251 cell migratory and invasive phenotype.

Expression of SKA2 is upregulated, and positively correlated with SPRY4-IT1 in glioma tissues. The correlation of SPRY4-IT1 and SKA2 expression was assessed in glioma samples. To identify the aberrant expression of SKA2 in glioma tissues further, RT-qPCR was used. Similar to the expression of SPRY4-IT1, the expression of SKA2 was much higher in glioma tissues than in normal brain tissues (Fig. 3A). The expression of SKA2 was higher in high-grade glioma compared with low-grade glioma (Fig. 3B). Notably, Pearson's correlation analysis demonstrated that the expression of SPRY4-IT1 was positively correlated with that of SKA2 in glioma tissues, which further indicates that SPRY4-IT1 is associated with the expression of SKA2 (Fig. 3C).

To determine the role of SPRY4-IT1 in SKA2 expression, U251 cells were transfected with si-SPRY4-IT1. U251 cells transfected with si-SPRY4-IT1 expressed lower levels of SKA2 mRNA than cells transfected with si-NC (Fig. 3D). Similarly, the protein expression of SKA2 was significantly reduced in si-SPRY4-IT1 cells compared with si-NC U251 cells (Fig. 3C). These data indicated that SPRY4-IT1 promotes SKA2 expression in U251 cells.
Knockdown of SKA2 inhibits the biological behavior of U251 cells. To investigate the role of SKA2 on U251 cells, SKA2 expression was knocked down. SKA2 expression was markedly decreased in U251 cells following transfection of si-SKA2 compared with si-NC (Fig. 4A and B). Similar to results observed with SPRY4-IT1-knockdown, knockdown of SKA2 significantly inhibited U251 cell proliferation and colony formation (Fig. 4C and D). Additionally, the invasion of U251 cells was inhibited in si-SKA2 cells compared with si-NC cells (Fig. 4E). To investigate the mechanism by which SKA2-knockdown inhibited formation of the glioma malignant phenotype, investigated the effect of SKA2 on the expression of proteins involved in cellular proliferation, including cyclin D1 and PCNA, and invasion, including MMP2 and MMP9. Knockdown of SKA2 reduced the expression of cyclin D1 and PCNA in U251 cells compared with si-NC cells, whereas the expression of MMP2 and MMP9 was significantly reduced in the cells transfected with si-SKA2 siRNA (Fig. 5A). Together, these results indicate that the knockdown of SKA2 inhibits cell proliferation, which is associated with the decreased expression of cyclin D1 and PCNA, and suppresses the invasive phenotype associated with the reduced expression levels of MMP2 and MMP9 in U251 cells. 

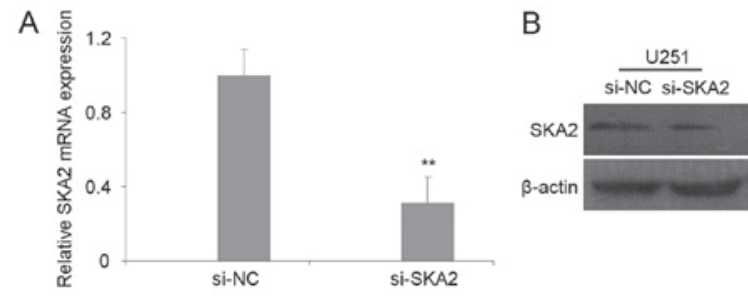

C 120
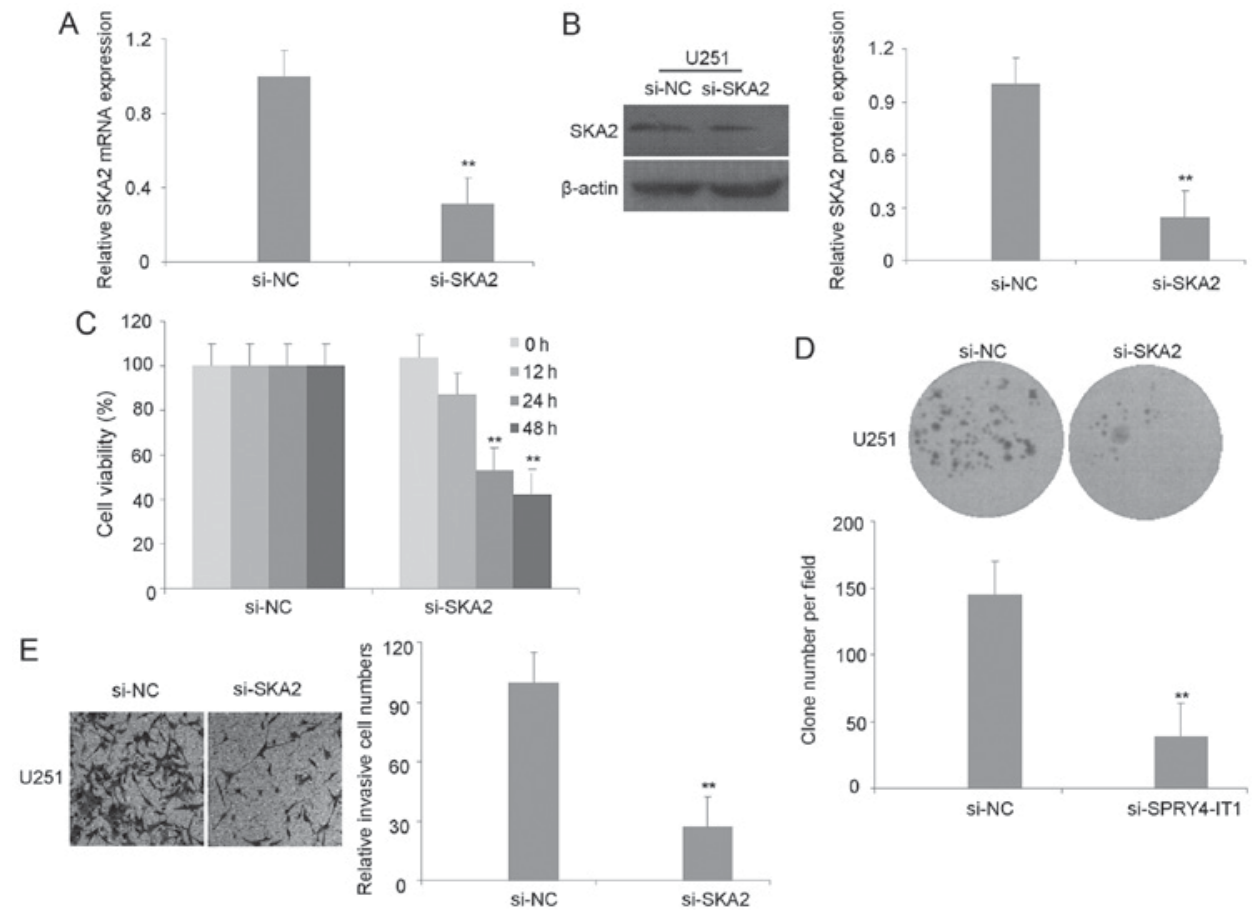

D

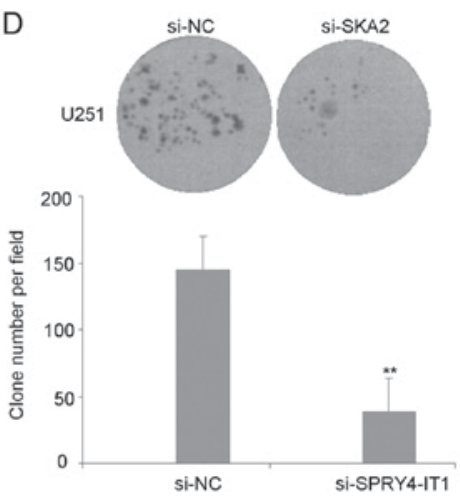

Figure 4. Knockdown of SKA2 inhibits cell growth in U251 cells. (A) Reverse transcription-quantitative polymerase chain reaction analysis of SKA2 mRNA expression in si-SKA2 and si-NC. (B) Western blot analysis of SKA2 protein expression in si-SKA2 and si-NC. (C) U251 cell proliferation in the si-SKA2 groups was significantly reduced, as detected by the MTT assay. (D) Effects of SKA2 on clone formation in U251 cells. (E) Effects of SKA2 on the invasion of U251 cells. ${ }^{* *} \mathrm{P}<0.01$ vs. si-NC. SKA2, spindle and kinetochore associated complex subunit 2; si-NC, U251 cells transfected with negative control small interfering RNA.
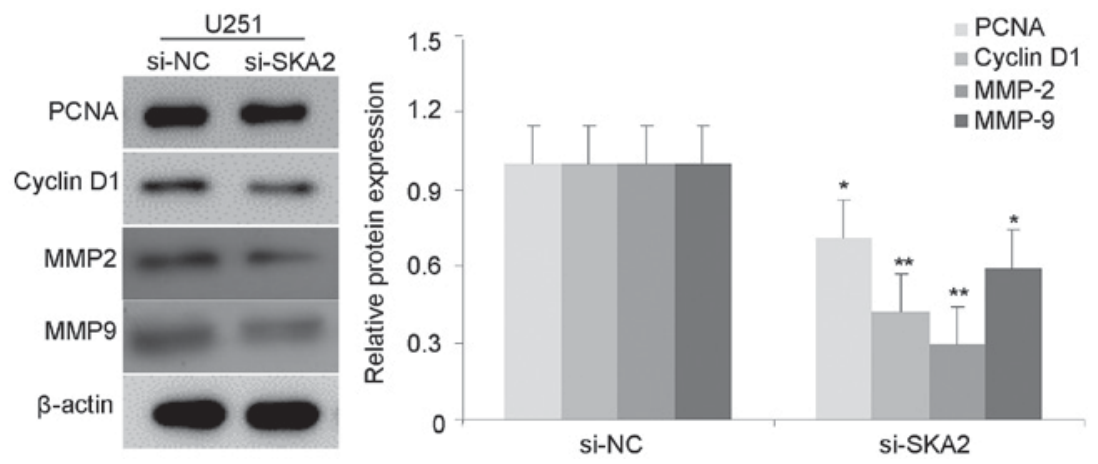

Figure 5. Effect of SKA2 on the expression of PCNA and cyclin D1 in U251 cells. Western blotting was performed to assess the protein level of PCNA, cyclin D1, MMP2 and MMP9 in siRNA-treated U251 cells. ${ }^{*} \mathrm{P}<0.05,{ }^{* *} \mathrm{P}<0.01$ vs. si-NC. SKA2, spindle and kinetochore associated complex subunit 2 ; PCNA, proliferating cell nuclear antigen; MMP2, matrix metalloproteinase-2; siRNA, small interfering RNA; si-NC, U251 cells transfected with negative control siRNA.

\section{Discussion}

Glioma is the most common primary malignancy of the human brain (22). Despite the use of aggressive therapeutic strategies, including surgical resection, radiotherapy and chemotherapy, the prognosis of glioma patients remains poor (23). Previous studies demonstrated that glioma development is associated with rates of cellular proliferation, migration, apoptosis and invasion $(1,24)$. It is therefore vital to understand the major regulatory mechanisms of this malignancy, which is key to the development of novel and effective therapeutic interventions for gliomas.

IncRNAs are a class of RNA molecules that regulate the transcription of target genes, meaning that the difference in IncRNA profiling between cancer and normal cells may indicate the mechanisms of cancer transformation. Evidence indicates that lncRNAs may serve pivotal roles in a range of cancer-associated biological processes, including in glioma $(15,16)$. By acting as oncogenes or tumor suppressors, IncRNAs have the potential to contribute to glioma initiation, progression and other malignant phenotypes. SPRY4-IT1 is a lncRNA that was initially associated with melanoma, and is upregulated in melanoma patient samples (17). The present study investigated SPRY4-IT1 expression in gliomas patients for the first time. SPRY4-IT1 expression was upregulated in gliomas tissues and cell lines compared with normal brain tissues. However, SPRY4-IT1 expression was markedly downregulated in non-small cell lung cancer tissues, compared with normal tissues (25). These findings indicate that SPRY4-IT1 expression may be tissue- and cell-specific, and that SPRY4-IT1 
may serve different roles in different tissues and stages of life process.

A previous study demonstrated that SPRY4-IT1 regulated the cell growth in melanoma cells; siRNA-mediated SPRY4-IT1-knockdown in melanoma cells resulted in the inhibition of cell growth, and an increase in the rate of apoptosis (17). Xie et al (26) demonstrated that knockdown of SPRY4-IT1 decreased the growth of esophageal squamous cell carcinoma in a xenograft mice model. The present study demonstrated that knockdown of SPRY4-IT1 significantly decreased the growth of U251 cells in vitro. Overexpression of SPRY4-IT1 has been shown to promote melanoma cell migration and invasiveness, whereas knockdown of SPRY4-IT1 inhibits melanoma cell invasion (17). The present study revealed that knockdown of SPRY4-IT1 inhibited the migration and invasion of U251 cells; however, this phenomenon was not consistent with the study reported by Zou et al conducted in trophoblast cells (27). Knockdown of SPRY4-IT1 induced the migration of HTR-8/SVneo cells, with overexpression of SPRY4-IT1 resulting in the inhibition of this migration (28). These findings indicate that lncRNA may present different biological functions in different cancer cells. For example, overexpression of HOTAIR results in an increase in the invasiveness and metastasis in primary breast tumors (29). Accordingly, knockdown of HOTAIR inhibited cell proliferation and induced apoptosis in pancreatic cancer cells (28). Although only a few functional lncRNAs have been well identified, they have been found to regulate gene expression at the levels of transcription, post-transcription and chromatin modification $(30,31)$.

SKA2 has been reported to locate to the kinetochore-microtubule interface during mitosis, whereas SKA-depleted cells cause chromosome congression defects and subsequently cell death $(18,32)$. A prior study revealed that A549 cells were treated with a microRNA-301 inhibitor or SKA2-specific siRNA, the mitotic index of the cells significantly increased, with a corresponding decrease in the colony formation ability (33). Overexpression of SKA2 appears to promote proliferation and human breast cancer progression (34). Recently, microarray analysis to identify genes that exhibited a change in expression following SPRY4-IT1 knockdown in MDA-MB-231 cells, indicating that SKA2 may represent a notable downstream effector of SPRY4-IT1 (35). The present study revealed that SKA2 expressions were elevated in glioma tissues; a significant positive correlation was also observed between SPRY4-IT1 and SKA2 in human glioma tissues. An in vitro experiment confirmed that SPRY4-IT1 knockdown markedly reduced SKA2 expression in U251 cells. However, the biological function of SKA2 in glioma remains unknown. The present study provides evidence that SKA2 serves an oncogenic role in U251 cells. SKA2 knockdown suppressed cellular proliferation and the invasiveness of U251 cells. The mechanism behind these behaviors may be associated with the decreased expression of proteins associated with proliferation and invasion. However, the precise molecular mechanism of how SPRY4-IT1 regulates SKA2 expression remains unclear and requires further study.

In conclusion, to the best of our knowledge for the first time, the present study demonstrated that SPRY4-IT1 expression was increased in glioma tissues, whereas the knockdown of SPRY4-IT1 inhibited U251 cells growth, migration and invasion. SPRY4-IT1 may control the glioma malignant phenotype via regulation of SKA2 expression. Further investigation of the functional and clinical implications of SPRY4-IT1 and its target SKA2 may facilitate the identification of novel therapeutic targets for glioma.

\section{Acknowledgements}

This study was supported by grants from the National Natural Science Foundation of China (nos. 81402078 and 81502149) and the Natural Science Foundation of Anhui Province (nos. 1608085MH225 and 1508085MH194).

\section{References}

1. Hamza MA and Gilbert M: Targeted therapy in gliomas. Curr Oncol Rep 16: 379, 2014.

2. De Witt Hamer PC, Robles SG, Zwinderman AH, Duffau H and Berger MS: Impact of intraoperative stimulation brain mapping on glioma surgery outcome: A meta-analysis. J Clin Oncol 30: 2559-2565, 2012

3. Smith JS, Chang EF, Lamborn KR, Chang SM, Prados MD, Cha S, Tihan T, Vandenberg S, McDermott MW and Berger MS: Role of extent of resection in the long-term outcome of low-grade hemispheric gliomas. J Clin Oncol 26: 1338-1345, 2008 .

4. van den Bent MJ, Afra D, de Witte O, Ben Hassel M, Schraub S, Hoang-Xuan K, Malmström PO, Collette L, Piérart M, Mirimanoff R, et al: Long-term efficacy of early versus delayed radiotherapy for low-grade astrocytoma and oligodendroglioma in adults: The EORTC 22845 randomised trial. Lancet 366: 985-990, 2005

5. Carninci P, Kasukawa T, Katayama S, Gough J, Frith MC, Maeda N, Oyama R, Ravasi T, Lenhard B, Wells C, et al: The transcriptional landscape of the mammalian genome. Science 309: 1559-1563, 2005.

6. Trapnell C, Williams BA, Pertea G, Mortazavi A, Kwan G, van Baren MJ, Salzberg SL, Wold BJ and Pachter L: Transcript assembly and quantification by RNA-Seq reveals unannotated transcripts and isoform switching during cell differentiation. Nat Biotechnol 28: 511-515, 2010.

7. Bartel DP: MicroRNAs: Genomics, biogenesis, mechanism, and function. Cell 116: 281-297, 2004.

8. Farazi TA, Spitzer JI, Morozov P and Tuschl T: miRNAs in human cancer. J Pathol 223: 102-115, 2011.

9. Louro R, Smirnova AS and Verjovski-Almeida S: Long intronic noncoding RNA transcription: Expression noise or expression choice? Genomics 93: 291-298, 2009.

10. Nagano $T$ and Fraser P: No-nonsense functions for long noncoding RNAs. Cell 145: 178-181, 2011.

11. Wapinski $\mathrm{O}$ and Chang HY: Long noncoding RNAs and human disease. Trends Cell Biol 21: 354-361, 2011.

12. Pandey RR, Mondal T, Mohammad F, Enroth S, Redrup L, Komorowski J, Nagano T, Mancini-Dinardo D and Kanduri C: Kcnq1ot1 antisense noncoding RNA mediates lineage-specific transcriptional silencing through chromatin-level regulation. Mol Cell 32: 232-246, 2008.

13. Wang X, Arai S, Song X, Reichart D, Du K, Pascual G, Tempst P, Rosenfeld MG, Glass CK and Kurokawa R: Induced ncRNAs allosterically modify RNA-binding proteins in cis to inhibit transcription. Nature 454: 126-130, 2008.

14. Zhao J, Sun BK, Erwin JA, Song JJ and Lee JT: Polycomb proteins targeted by a short repeat RNA to the mouse X chromosome. Science 322: 750-756, 2008.

15. Wang P, Ren Z and Sun P: Overexpression of the long non-coding RNA MEG3 impairs in vitro glioma cell proliferation. J Cell Biochem 113: 1868-1874, 2012.

16. Zhang JX, Han L, Bao ZS, Wang YY, Chen LY, Yan W, Yu SZ, Pu PY, Liu N, You YP, et al: HOTAIR, a cell cycle-associated long noncoding RNA and a strong predictor of survival, is preferentially expressed in classical and mesenchymal glioma. Neuro Oncol 15: 1595-1603, 2013. 
17. Khaitan D, Dinger ME, Mazar J, Crawford J, Smith MA, Mattick JS and Perera RJ: The melanoma-upregulated long noncoding RNA SPRY4-IT1 modulates apoptosis and invasion. Cancer Res 71: 3852-3862, 2011.

18. Hanisch A, Sillje HH and Nigg EA: Timely anaphase onset requires a novel spindle and kinetochore complex comprising Ska1 and Ska2. EMBO J 25: 5504-5515, 2006.

19. Rice L, Waters CE, Eccles J, Garside H, Sommer P, Kay P, Blackhall FH, Zeef L, Telfer B, Stratford I, et al: Identification and functional analysis of SKA2 interaction with the glucocorticoid receptor. J Endocrinol 198: 499-509, 2008.

20. Louis DN, Ohgaki H, Wiestler OD, Cavenee WK, Burger PC, Jouvet A, Scheithauer BW and Kleihues P: The 2007 WHO classification of tumours of the central nervous system. Acta Neuropathol 114: 97-109, 2007.

21. Livak KJ and Schmittgen TD: Analysis of relative gene expression data using real-time quantitative PCR and the 2(-Delta Delta C(T)). Methods 25: 402-408, 2001.

22. Furnari FB, Fenton T, Bachoo RM, Mukasa A, Stommel JM, Stegh A, Hahn WC, Ligon KL, Louis DN, Brennan C, et al: Malignant astrocytic glioma: Genetics, biology, and paths to treatment. Genes Dev 21: 2683-2710, 2007.

23. He J, Zhang W, Zhou Q, Zhao T, Song Y, Chai L and Li Y: Low-expression of microRNA-107 inhibits cell apoptosis in glioma by upregulation of SALL4. Int J Biochem Cell Biol 45: 1962-1973, 2013.

24. Diamandis P and Aldape KD: Insights from molecular profiling of adult glioma. J Clin Oncol 35: 2386-2393, 2017.

25. Sun M, Liu XH, Lu KH, Nie FQ, Xia R, Kong R, Yang JS, Xu TP Liu YW, Zou YF, et al: EZH2-mediated epigenetic suppression of long noncoding RNA SPRY4-IT1 promotes NSCLC cell proliferation and metastasis by affecting the epithelial-mesenchymal transition. Cell Death Dis 5: e1298, 2014.

26. Xie HW, Wu QQ, Zhu B, Chen FJ, Ji L, Li SQ, Wang CM, Tong YS, Tuo L, Wu M, et al: Long noncoding RNA SPRY4-IT1 is upregulated in esophageal squamous cell carcinoma and associated with poor prognosis. Tumour Biol 35: 7743-7754, 2014.
27. Zou Y, Jiang Z, Yu X, Sun M, Zhang Y, Zuo Q, Zhou J, Yang N, Han P, Ge Z, et al: Upregulation of long noncoding RNA SPRY4-IT1 modulates proliferation, migration, apoptosis, and network formation in trophoblast cells HTR-8SV/neo. PLoS One 8: e79598, 2013.

28. Gupta RA, Shah N, Wang KC, Kim J, Horlings HM, Wong DJ, Tsai MC, Hung T, Argani P, Rinn JL, et al: Long non-coding RNA HOTAIR reprograms chromatin state to promote cancer metastasis. Nature 464: 1071-1076, 2010.

29. Kim K, Jutooru I, Chadalapaka G, Johnson G, Frank J, Burghardt R, Kim S and Safe S: HOTAIR is a negative prognostic factor and exhibits pro-oncogenic activity in pancreatic cancer. Oncogene 32: 1616-1625, 2013.

30. Ponting CP, Oliver PL and Reik W: Evolution and functions of long noncoding RNAs. Cell 136: 629-641, 2009.

31. Tsai MC, Manor O, Wan Y, Mosammaparast N, Wang JK, Lan F, Shi Y, Segal E and Chang HY: Long noncoding RNA as modular scaffold of histone modification complexes. Science 329: 689-693, 2010

32. Gaitanos TN, Santamaria A, Jeyaprakash AA, Wang B, Conti E and Nigg EA: Stable kinetochore-microtubule interactions depend on the Ska complex and its new component Ska3/C13Orf3. EMBO J 28: 1442-1452, 2009

33. Cao G, Huang B, Liu Z, Zhang J, Xu H, Xia W, Li J, Li S, Chen L, Ding H, et al: Intronic miR-301 feedback regulates its host gene, ska2, in A549 cells by targeting MEOX2 to affect ERK/CREB pathways. Biochem Biophys Res Commun 396: 978-982, 2010

34. Shi W, Gerster K, Alajez NM, Tsang J, Waldron L, Pintilie M, Hui AB, Sykes J, P'ng C, Miller N, et al: MicroRNA-301 mediates proliferation and invasion in human breast cancer. Cancer Res 71: 2926-2937, 2011.

35. Shi Y, Li J, Liu Y, Ding J, Fan Y, Tian Y, Wang L, Lian Y, Wang K and Shu Y: The long noncoding RNA SPRY4-IT1 increases the proliferation of human breast cancer cells by upregulating ZNF703 expression. Mol Cancer 14: 51, 2015. 